Pacific Journal of Mathematics

THE ISOMORPHISM PROBLEM FOR ORTHODOX 


\title{
THE ISOMORPHISM PROBLEM FOR ORTHODOX SEMIGROUPS
}

\author{
T. E. HALL
}

The author's structure theorem for orthodox semigroups produced an orthodox semigroup $\mathscr{H}(E, T, \psi)$ from a band $E$, an inverse semigroup $T$ and a morphism $\psi$ between two inverse semigroups, namely $T$ and $W_{E} / \gamma$, an inverse semigroup constructed from $E$. Here, we solve the isomorphism problem: when are two such orthodox semigroups isomorphic? This leads to a way of producing all orthodox semigroups, up to isomorphism, with prescribed band $E$ and maximum inverse semigroup morphic image $T$.

1. Preliminaries. A semigroup $S$ is called regular (in the sense of von Neumann for rings) if for each $a \in S$ there exists $x \in S$ such that $a x a=a$; and $S$ is called an inverse semigroup if for each $a \in S$ there is' a unique $x \in S$ such that $a x a=a$ and $x a x=x$. A band is a semigroup in which each element is idempotent, and an orthodox semigroup is a regular semigroup in which the idempotents form a subsemigroup (that is, a band).

We follow the notation and conventions of Howie [4].

Result 1 [3, Theorem 5]. The maximum congruence contained in Green's relation $\mathscr{H}$ on any regular semigroup $S, \mu=\mu(S)$ say, is given by $\mu=\left\{(a, b) \in \mathscr{H}:\right.$ for some [for each pair of ] $\mathscr{H}$-related inverses $a^{\prime}$ of $a$ and $b^{\prime}$ of $b, a^{\prime} e a=b^{\prime} e b$ for each idempotent $\left.e \leq a a^{\prime}\right\}$.

A regular semigroup $S$ is called fundamental if $\mu$ is the identity relation on $S$. For each band $E$, the semigroup $W_{E}$ is fundamental, orthodox, has its band isomorphic to $E$, and contains, for each orthodox semigroup $S$ with band $E$, a copy of $S / \mu$ as a subsemigroup: see the author [1] (or [3] with $E=\langle E\rangle$ and $W_{E}=T_{\langle E\rangle}$ ) or Howie [4, $\S$ VI.2].

Now take any inverse semigroup $T$, and, if such exist, any idempotent-separating morphism $\psi: T \rightarrow W_{E} / \gamma$ whose range contains the semilattice of all idempotents of $W_{E} / \gamma$, where $\gamma$ denotes the least inverse semigroup congruence on $W_{E}$. A semigroup $\mathscr{H}(E, T, \psi)$ (see 
$S(E, T, \psi)$ in the author [2], or see Howie [4, §VI.4]) is defined by

$$
\mathscr{H}(E, T, \psi)=\left\{(w, t) \in W_{E} \times T: w \gamma^{\natural}=t \psi\right\} ;
$$

that is, $\mathscr{H}(E, T, \psi)$ occurs in the pullback diagram

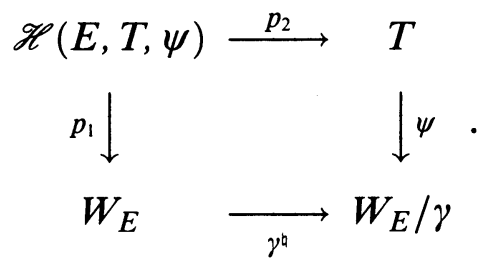

Here, $p_{1}$ and $p_{2}$ are projections.

The semigroup $\mathscr{H}(E, T, \psi)$ is orthodox, has band isomorphic to $E$, and has its maximum inverse semigroup morphic image isomorphic to $T$; conversely every such semigroup is obtained in this way (the author [2], or Howie [4, §VI.4]).

\section{The isomorphism problem.}

LEMMA 1. Take any two morphisms $\varphi, \psi$ from a regular semigroup $T$ to a regular semigroup $S$ such that the range of each of $\varphi$ and $\psi$ contains the set $E(S)$ of all the idempotents of $S$. If $\varphi|E(T)=\psi| E(T)$ then $(t \varphi, t \psi) \in \mu$, for all $t \in T$; in particular, if also $S$ is fundamental, then $\varphi=\psi$.

Proof. Take any $t \in T$ and any inverse $t^{\prime}$ of $t$ in $T$. Of course, in $S, t^{\prime} \varphi$ and $t^{\prime} \psi$ are inverses of $t \varphi$ and $t \psi$ respectively and $\left(t^{\prime} \varphi\right)(t \varphi)=$ $\left(t^{\prime} t\right) \varphi=\left(t^{\prime} t\right) \psi=\left(t^{\prime} \psi\right)(t \psi)$. Likewise $(t \varphi)\left(t^{\prime} \varphi\right)=(t \psi)\left(t^{\prime} \psi\right)$, so $(t \varphi) \mathscr{H}(t \psi)$ and $\left(t^{\prime} \varphi\right) \mathscr{H}\left(t^{\prime} \psi\right)$. Take any idempotent $e$ of $S$ such that $e \leq\left(t t^{\prime}\right) \varphi$ and any $x \in T$ such that $x \varphi=e$ : then $\left(t t^{\prime} x t t^{\prime}\right) \varphi=$ $\left[\left(t t^{\prime}\right) \varphi\right] e\left[\left(t t^{\prime}\right) \varphi\right]=e$, so $e \in \operatorname{range}\left(\varphi \mid t t^{\prime} T t t^{\prime}\right)$. Now $t t^{\prime} T t t^{\prime}$ is a regular semigroup, so by Lallement's Lemma [4, Lemma II.4.7] there is an idempotent $f \in t t^{\prime} T t t^{\prime}$ such that $f \varphi=e$. Since $t^{\prime} f t$ is idempotent, we have

$$
\begin{aligned}
\left(t^{\prime} \varphi\right) e(t \varphi) & =\left(t^{\prime} \varphi\right)(f \varphi)(t \varphi)=\left(t^{\prime} f t\right) \varphi=\left(t^{\prime} f t\right) \psi \\
& =\left(t^{\prime} \psi\right)(f \psi)(t \psi)=\left(t^{\prime} \psi\right) e(t \psi) .
\end{aligned}
$$

Thus $(t \varphi, t \psi) \in \mu$, as required, completing the proof.

Take any isomorphism $\alpha: E \rightarrow E^{\prime}$ from a band $E$ to a band $E^{\prime}$. Consider $W_{E}$ and $W_{E^{\prime}}$ and, as usual, identify $E$ and $E^{\prime}$ with the bands of 
$W_{E}$ and $W_{E^{\prime}}$ respectively. Since $W_{E^{\prime}}$ is constructed from $E^{\prime}$ precisely as $W_{E}$ is constructed from $E$, there is an isomorphism from $W_{E}$ to $W_{E^{\prime}}$ extending $\alpha$, say $\alpha^{*}$ (in fact, there is a unique such isomorphism, by Lemma 1). Denote by $\gamma$ and $\gamma^{\prime}$ the least inverse semigroup congruences on $W_{E}$ and $W_{E^{\prime}}$ respectively: then the map $\alpha^{* *}: W_{E} / \gamma \rightarrow W_{E^{\prime}} / \gamma^{\prime}$, given by $w \gamma \alpha^{* *}=w \alpha^{*} \gamma^{\prime}$, for all $w \in W_{E}$, is an isomorphism such that $\gamma^{\natural} \alpha^{* *}=\alpha^{*} \gamma^{\prime}$, and is the unique such isomorphism. Summarizing, we have that the following diagram commutes, and $\alpha^{*}, \alpha^{* *}$ are the unique morphisms making the diagram commute.

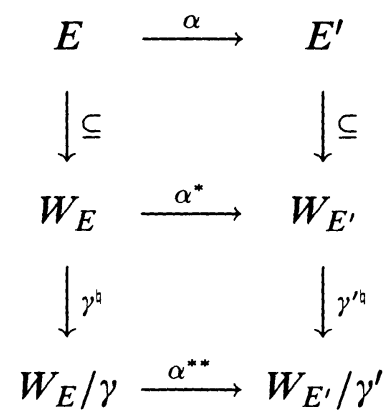

THEOREM 2. Take any bands $E, E^{\prime}$, inverse semigroups $T, T^{\prime}$ and idempotent-separating morphisms $\psi: T \rightarrow W_{E} / \gamma$ and $\psi^{\prime}: T^{\prime} \rightarrow W_{E^{\prime}} / \gamma^{\prime}$ whose ranges contain the idempotents of $W_{E} / \gamma$ and $W_{E^{\prime}} / \gamma^{\prime}$ respectively. Then $\mathscr{H}(E, T, \psi)$ is isomorphic to $\mathscr{H}\left(E^{\prime}, T^{\prime}, \psi^{\prime}\right)$ if and only if there exist isomorphisms $\alpha: E \rightarrow E^{\prime}$ and $\beta: T \rightarrow T^{\prime}$ such that the following diagram commutes

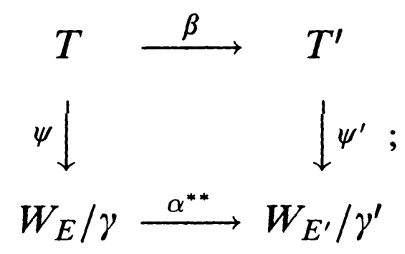

that is, such that $\psi^{\prime}=\beta^{-1} \psi \alpha^{* *}$.

Proof. (a) if statement. Suppose such $\alpha, \beta$ exist. Informally we could say that $E^{\prime}, T^{\prime}, \psi^{\prime}$ are a renaming of $E, T, \psi$ respectively, obtained by renaming each $e \in E$ by $e \alpha$ and each $t \in T$ by $t \beta$, and so $\mathscr{H}\left(E^{\prime}, T^{\prime}, \psi^{\prime}\right)$ is isomorphic to $\mathscr{H}(E, T, \psi)$. More formally, we consider the isomorphism $\left(\alpha^{*}, \beta\right): W_{E} \times T \rightarrow W_{E^{\prime}} \times T^{\prime}$ given by $(w, t)\left(\alpha^{*}, \beta\right)=\left(w \alpha^{*}, t \beta\right)$ for all $(w, t) \in W_{E} \times T$, and we show that $\mathscr{H}(E, T, \psi)\left(\alpha^{*}, \beta\right)=\mathscr{H}\left(E^{\prime}, T^{\prime}, \psi^{\prime}\right)$. 
Take any $(w, t) \in \mathscr{H}(E, T, \psi)$ : then $w \gamma^{\natural}=t \psi$, and so

$$
t \beta \psi^{\prime}=t \beta \beta^{-1} \psi \alpha^{* *}=t \psi \alpha^{* *}=w \gamma^{\natural} \alpha^{* *}=w \alpha^{*} \gamma^{\natural \natural},
$$

so $(w, t)\left(\alpha^{*}, \beta\right)=\left(w \alpha^{*}, t \beta\right) \in \mathscr{H}\left(E^{\prime}, T^{\prime}, \psi^{\prime}\right)$ and hence $\mathscr{H}(E, T, \psi)\left(\alpha^{*}, \beta\right)$ $\subseteq \mathscr{H}\left(E^{\prime}, T^{\prime}, \psi^{\prime}\right)$.

From symmetry, we deduce that

$$
\mathscr{H}\left(E^{\prime}, T^{\prime}, \psi^{\prime}\right)\left(\alpha^{*}, \beta\right)^{-1}=\mathscr{H}\left(E^{\prime}, T^{\prime}, \psi^{\prime}\right)\left(\alpha^{*-1}, \beta^{-1}\right) \subseteq \mathscr{H}(E, T, \psi),
$$

whence $\mathscr{H}(E, T, \psi)\left(\alpha^{*}, \beta\right)=\mathscr{H}\left(E^{\prime}, T^{\prime}, \psi^{\prime}\right)$, as required.

(b) only if statement. Informally, we could say that, for any orthodox semigroup $S$ with band $E$ and least inverse semigroup congruence $\mathscr{Y}$, there is a unique morphism $\psi$ making the following diagram commute:

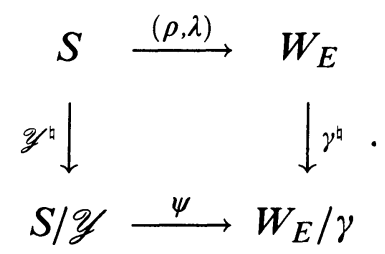

Hence $E, S / \mathscr{Y}, \psi$ are all determined to within isomorphisms (or renamings) by $S$. Formally, we proceed as follows.

Take any isomorphism $\theta: S \rightarrow S^{\prime}$, where $S=\mathscr{H}(E, T, \psi)$ and $S^{\prime}=\mathscr{H}\left(E^{\prime}, T^{\prime}, \psi^{\prime}\right)$. Put $\theta \mid E=\alpha$, an isomorphism of $E$ upon $E^{\prime}$, by Lallement's Lemma [4, Lemma II.4.7]. Let $\mathscr{Y}$ and $\mathscr{Y}^{\prime}$ denote the least inverse semigroup congruences on $S$ and $S^{\prime}$ respectively. Clearly there is a unique isomorphism $\beta: S / \mathscr{Y} \rightarrow S^{\prime} / \mathscr{Y}^{\prime}$ making the following diagram commute:

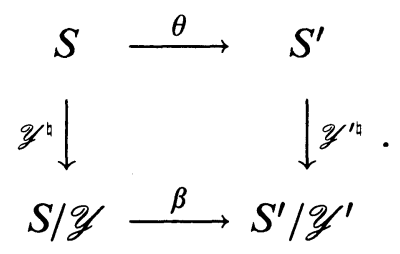

Now $T \cong S / \mathscr{Y}$ and $T^{\prime} \cong S^{\prime} / \mathscr{Y}^{\prime}$ ([2, Theorem 1] or [4, Theorem VI.4.6]), so we assume without loss of generality that $T=S / \mathscr{Y}$ and $T^{\prime}=S^{\prime} / \mathscr{Y}^{\prime}$; it remains to show that $\psi^{\prime}=\beta^{-1} \psi \alpha^{* *}$. 
We shall see that Diagram 1 commutes $\left(p_{1}, p_{2}, p_{1}^{\prime}, p_{2}^{\prime}\right.$ are projections).

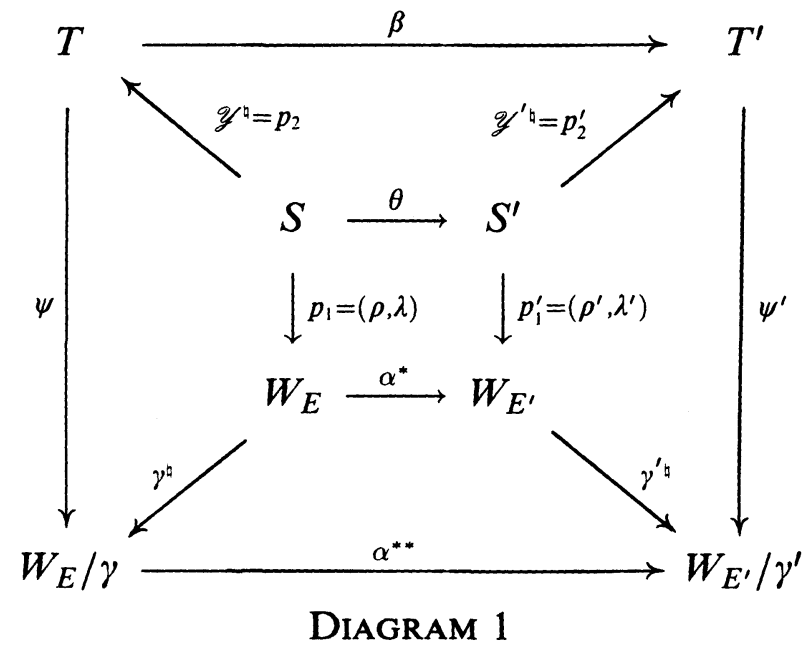

We have seen already that each of the four outer faces is a commuting diagram: we consider the central face. Now $\theta p_{1}^{\prime}$ and $p_{1} \alpha^{*}$ are morphisms which agree on $E$ (with $\alpha=\theta \mid E$ ), and which map $E$ (isomorphically) onto $E^{\prime}$, the band of $W_{E^{\prime}}$. Hence, by Lemma $1, \theta p_{1}^{\prime}=p_{1} \alpha^{*}$; that is, the central face commutes.

Consideration of the external face leads us to the following diagram.

$$
\begin{array}{r}
S \stackrel{p_{1}=(\rho, \lambda)}{\longrightarrow} W_{E} \\
\mathscr{Y}^{\natural}=p_{2} \downarrow \\
T \underset{\beta \psi^{\prime} \alpha^{* *-1}}{\stackrel{\psi}{\longrightarrow}} W_{E} / \gamma
\end{array}
$$

The commuting of the five internal faces of Diagram 1 gives us that $p_{1} \gamma^{\natural}=p_{2} \beta \psi^{\prime} \alpha^{* *-1}$. But the mapping $s \mathscr{Y} \mapsto\left(\rho_{S}, \lambda_{S}\right) \gamma$ (for all $s \in S$ ), namely $\psi$, is the unique morphism from $T$ to $W_{E} / \gamma$ making this diagram commute, and hence $\psi=\beta \psi^{\prime} \alpha^{* *-1}$ (that is, the external face commutes) and so $\psi^{\prime}=\beta^{-1} \psi \alpha^{* *}$ as required.

3. Orthodox semigroups, up to isomorphism. Consider the following problem: given a band $E$ and an inverse semigroup $T$, find, up to isomorphism, the orthodox semigroups with band $E$ and with maximum inverse semigroup morphic image isomorphic to $T$.

The author's structure theorem ([2, Theorem 1] or [4, Theorem VI.4.6]) and Theorem 2 above together immediately yield a solution as follows. 
Denote by $\operatorname{Aut}(S)$ the group of automorphisms of any semigroup $S$. From Lemma 1, for any $\varphi \in \operatorname{Aut}\left(W_{E}\right)$, we see that $\varphi=(\varphi \mid E)^{*}$, so we have that $\operatorname{Aut}(E) \cong \operatorname{Aut}\left(W_{E}\right)$ under the map $\alpha \mapsto \alpha^{*}$ for each $\alpha \in \operatorname{Aut}(E)$. The map $\operatorname{Aut}\left(W_{E}\right) \rightarrow \operatorname{Aut}\left(W_{E} / \gamma\right), \alpha^{*} \mapsto \alpha^{* *}$ (for each $\alpha \in \operatorname{Aut}(E))$, is a morphism; we denote its image by $[\operatorname{Aut}(E)]^{* *}$; then $[\operatorname{Aut}(E)]^{* *}=\left\{\alpha^{* *}: \alpha \in \operatorname{Aut}(E)\right\}$.

Denote by $M$ the set of idempotent-separating morphisms from $T$ into $W_{E} / \gamma$ whose ranges each contain the idempotents of $W_{E} / \gamma$. By [2, Corollary 1] or [4, Theorem VI.4.6], there exists an orthodox semigroup with band $E$ and with maximum inverse semigroup morphic image isomorphic to $T$, if and only if $M$ is nonempty. Assume henceforth that $M$ is nonempty. Define an action on $M$ by the group $\operatorname{Aut}(T) \times[\operatorname{Aut}(E)]^{* *}$ as follows:

$$
\psi\left(\beta, \alpha^{* *}\right)=\beta^{-1} \psi \alpha^{* *},
$$

for all $\psi \in M, \beta \in \operatorname{Aut}(T), \alpha \in \operatorname{Aut}(E)$.

The orbits of $M$ under $\operatorname{Aut}(T) \times[\operatorname{Aut}(E)]^{* *}$ are the sets

$$
\psi\left(\operatorname{Aut}(T) \times[\operatorname{Aut}(E)]^{* *}\right)=\left\{\beta^{-1} \psi \alpha^{* *}: \beta \in \operatorname{Aut}(T), \alpha \in \operatorname{Aut}(E)\right\},
$$

for each $\psi \in M$ (thus these sets partition $M$ ). By Theorem 2, we have, for all $\psi, \psi^{\prime} \in M, \mathscr{H}(E, T, \psi) \cong \mathscr{H}\left(E, T, \psi^{\prime}\right)$ if and only if $\psi$ and $\psi^{\prime}$ are in the same orbit. Thus, if $\left\{\psi_{i}: i \in I\right\}$ is a transversal of the set of orbits (that is, a selection of precisely one morphism from each orbit) then $\mathscr{H}\left(E, T, \psi_{i}\right), i \in I$, is a list of all the orthodox semigroups with band $E$ and maximum inverse semigroup morphic image isomorphic to $T$, and the semigroups are pairwise nonisomorphic.

\section{REFERENCES}

[1] T. E. Hall, On orthodox semigroups and uniform and antiuniform bands, J. Algebra, 16 (1970), 204-217.

[2] - Orthodox semigroups, Pacific J. Math., 39 (1971), 677-686.

[3] - On regular semigroups, J. Algebra, 24 (1973), 1-24.

[4] J. M. Howie, An Introduction to Semigroup Theory, L.M.S. Monographs, No. 7, Academic Press, London, New York, 1976.

Received July 21, 1987.

MONASH UNIVERSITY

Clayton, Victoria 3168

Australia 


\section{PACIFIC JOURNAL OF MATHEMATICS EDITORS}

\author{
V. S. VARADARAJAN \\ (Managing Editor) \\ University of California \\ Los Angeles, CA 90024 \\ HeRbert Clemens \\ University of Utah \\ Salt Lake City, UT 84112 \\ THOMAS ENRIGHT \\ University of California, San Diego \\ La Jolla, CA 92093
}

R. FINN

Stanford University

Stanford, CA 94305

HERMANN FLASCHKA

University of Arizona

Tucson, AZ 85721

VAUGHAN F. R. JONES

University of California

Berkeley, CA 94720

STEVEN KERCKHOFF

Stanford University

Stanford, CA 94305

\section{ROBION KIRBY}

University of California

Berkeley, CA 94720

C. C. MOORE

University of California

Berkeley, CA 94720

HAROLD STARK

University of California, San Diego

La Jolla, CA 92093

\section{ASSOCIATE EDITORS}
R. ARenS
E. F. BECKENBACH
B. H. NeumanN
F. WOLF
K. YOSHIDA (1906-1982)

\section{SUPPORTING INSTITUTIONS}
UNIVERSITY OF ARIZONA
UNIVERSITY OF OREGON
UNIVERSITY OF BRITISH COLUMBIA
UNIVERSITY OF SOUTHERN CALIFORNIA
CALIFORNIA INSTITUTE OF TECHNOLOGY
STANFORD UNIVERSITY
UNIVERSITY OF CALIFORNIA
MONTANA STATE UNIVERSITY
UNIVERSITY OF HAWAII
UNIVERSITY OF NEVADA, RENO
UNIVERSITY OF TOKYO
NEW MEXICO STATE UNIVERSITY
UNIVERSITY OF UTAH
OREGON STATE UNIVERSITY
WASHINGTON STATE UNIVERSITY
UNIVERSITY OF WASHINGTON 


\section{Pacific Journal of Mathematics}

\section{Vol. 136, No. $1 \quad$ November, 1989}

Robert Archbold and Frederic W. Shultz, Characterization of $C^{*}$-algebras with continuous trace by properties of their pure states $\ldots \ldots \ldots \ldots \ldots \ldots 1$

Shu Ping Chen and Roberto Triggiani, Proof of extensions of two conjectures on structural damping for elastic systems $\ldots \ldots \ldots \ldots \ldots \ldots$

Philip Throop Church and James Timourian, A nonlinear elliptic

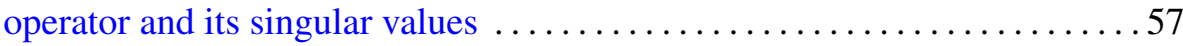

A. Gervasio Colares and Katsuei Kenmotsu, Isometric deformation of surfaces in $R^{3}$ preserving the mean curvature function $\ldots \ldots \ldots \ldots \ldots 71$

Fei Xu, A remark on spinor norms of local integral rotations. I . . . . . . . 81

Pedro Martinez Gadea and Ángel María Montesinos-Amilibia, Spaces of constant para-holomorphic sectional curvature $\ldots \ldots \ldots \ldots \ldots \ldots \ldots 5$

Guangxin Zeng, Homogeneous Stellensätze in semialgebraic geometry . . . . 103

Thomas Eric Hall, The isomorphism problem for orthodox semigroups . . . . 123

Mike Hoffman, Noncoincidence index, free group actions, and the fixed

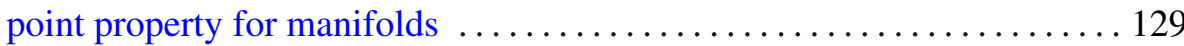

Terry Atherton Loring, The noncommutative topology of one-dimensional

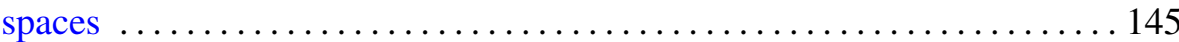

Haskell Paul Rosenthal and Alan Evan Wessel, The Krě̆ n-Mil'man property and a martingale coordinatization of certain nondentable convex sets

Yoshimi Saito, A remark on the limiting absorption principle for the reduced wave equation with two unbounded media 